
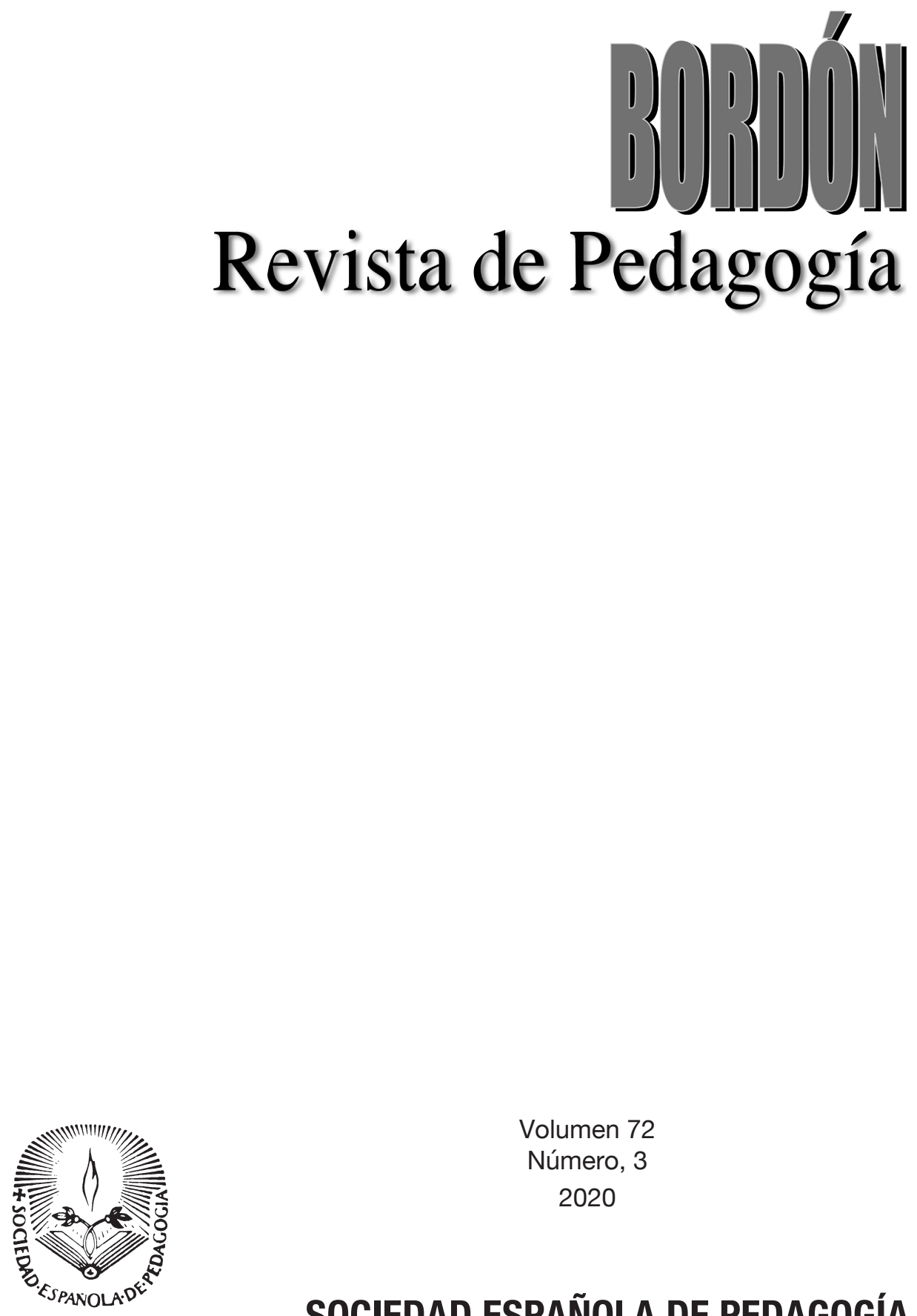

Volumen 72

Número, 3

2020 


\section{CAPITHLISMO ACADÉmICO: DISTIICIONES CONCEPTUALES Y PROCESOS CONTRADICTORIOS A PROPÓSITO DEL CASO CHILENO}

\section{Academic capitalism: conceptual distinctions and contradictory processes in reference to the Chilean case}

JOSÉ JOAQUíN BRUNNER(1), LILIANA PEDRAJA-REJAS( ${ }^{(2)}$ Y JULIO LABRAÑA VARGAS(1)

(I) Centro de Políticas Comparadas de Educación, Universidad Diego Portales (Chile)

(2) Universidad de Tarapacá (Chile)

D01: 10.13042/Bordon.2020.72761

Fecha de recepción: 24/05/2019 • Fecha de aceptación: 03/06/2020

Autor de contacto / Corresponding author: Julio Labraña Vargas. E-mail: julio.labrana@mail.udp.cl

INTRODUCCIÓN. El presente artículo revisa la literatura sobre la teoría del capitalismo académico, busca precisar y desarrollar sus conceptos básicos y luego los utiliza para analizar el caso chileno, tanto desde el punto de vista de la economía política de su sistema nacional de educación superior como de las transformaciones que experimentan las universidades en dirección a un comportamiento emprendedor y una gestión de tipo managerial. MÉTODO. Se realiza un análisis bibliométrico de la literatura especializada sobre capitalismo académico en inglés, español y portugués y se efectúa un análisis de contenido con el objetivo de identificar las distintas comprensiones del capitalismo académico. RESULTADOS. Se identifican dos definiciones de capitalismo académico: una enfocada en cambios en el nivel de la economía política de los sistemas y otra centrada en las transformaciones a nivel organizacional. DISCUSIÓN. Sobre esta base, se propone un marco analítico para estudiar el capitalismo académico, distinguiendo entre procesos de economía política (mercadización, privatización, mercantilización, comercialización y financiarización) y sus efectos organizacionales (universidad emprendedora y gerencialismo de la gestión). Este marco se usa para estudiar la reforma de la educación superior intentada en Chile entre 2014 y 2018, destinada a revertir o mitigar el avanzado grado de privatismo de su sistema, e identifica las transformaciones experimentadas por el capitalismo académico como resultado de esta reforma, concluyendo que los resultados en tal sentido son limitados y en algunos casos ambiguos, pudiendo incluso causar efectos no anticipados y contrarios al objetivo buscado. El artículo concluye con un resumen y posibles líneas futuras de investigación en esta área.

Palabras clave: Educación superior, Universidades, Educación postsecundaria como campo de estudio, Objetivos educacionales. 


\section{Introducción}

Aunque se ha establecido que el campo de investigación de la educación superior carece de un desarrollo consistente de teorías (Tight, 2019), durante las últimas décadas se han registrado avances en este ámbito (Bastedo, 2012). Un ejemplo es la teoría del capitalismo académico (Slaughter y Leslie, 1997; Slaughter y Rhoades, 2004), cuya difusión ha sido amplia en países del norte (Cantwell y Kauppinen, 2014), si bien circunscrita al idioma inglés y referida principalmente a las grandes universidades públicas de investigación de los Estados Unidos de América. En este artículo exploramos la polisemia del concepto de "capitalismo académico" en la literatura especializada y presentamos un marco analítico para analizar sus distintas dimensiones, el cual se usa a continuación para observar el desarrollo reciente de la educación superior chilena.

En efecto, el sistema chileno se halla envuelto en intensas dinámicas de privatismo (Brunner, 2017); esto es, un conjunto de procesos interrelacionados que amplifican las dinámicas privadas dentro del sistema y redefinen constantemente el rol del Estado frente a las universidades. Siguiendo el argumento original de Slaughter y Leslie (1997) y Slaughter y Rhoades (2004), dichos procesos favorecen la institucionalización de un régimen capitalista de conocimiento y aprendizaje. Sostenemos que la observación - desde el marco aquí propuesto- de las dinámicas que llevan a esa institucionalización enriquece la descripción del capitalismo académico y permite profundizar en las particularidades del caso chileno.

El artículo está organizado en las siguientes secciones: sobre la base de un análisis bibliométrico de la literatura sobre capitalismo académico (1), se propone un marco conceptual para identificar sus principales procesos de economía política - mercadización, privatización de la provisión y el financiamiento, mercantilización, comercialización y financiarización- y sus efectos a nivel organizacional, que son la empresarialización y el gerencialismo (2). Seguidamente se aplica dicho marco al caso chileno, extendiéndolo al estudio del conjunto reciente de políticas que buscan revertir las dinámicas del capitalismo académico en este país e interrogando sus efectos potenciales (3). El artículo concluye con un resumen y líneas de investigación de las distintas dimensiones que caracterizan al capitalismo académico (4).

\section{La comprensión del capitalismo académico: definiciones divergentes}

El análisis de la literatura sobre el capitalismo académico muestra que no existe una definición unívoca de este fenómeno. Para el presente estudio se utilizó el programa Publish or Perish para determinar los documentos registrados por Google Scholar que en su título contuvieran las palabras academic capitalism en inglés, español o portugués. El uso de Google Scholar se justifica, pues permite alcanzar la máxima cobertura posible de documentos académicos en una amplia gama de soportes (Harzing y Van der Wal, 2008). Estas búsquedas se realizaron en la segunda quincena del mes de mayo de 2018 y arrojaron 368 textos en inglés y 76 en español y portugués. Ambos listados fueron luego depurados, fusionando entradas duplicadas, corrigiendo autores o títulos mal identificados y eliminando entradas en idiomas distintos al inglés, español y portugués.

En idioma inglés el corpus se restringió a los documentos incluidos dentro del índice $\mathrm{H}$ de dicha base; esto es, aquellos con 33 citas o más, siguiendo el procedimiento especificado por Martínez, Herrera, López-Gijón y Herrera-Viedma (2014) para el estudio de autores que denominan $\mathrm{H}$ a los clásicos de una determinada área. A esta primera selección se agregaron los demás documentos pertenecientes a autores incluidos en la recopilación previa publicados dentro del quinquenio 2013-2018 con un número menor de citas que el respectivo índice $\mathrm{H}$, con el objeto 
Capitalismo académico: distinciones conceptuales y procesos contradictorios a propósito del caso chileno

de incluir así su producción más reciente en este estudio. Con esto, la muestra en idioma inglés se incrementó a 41 documentos. Para los documentos en español y portugués se siguió un procedimiento diferente, puesto que el índice $\mathrm{H}$ de este corpus resultó bajo (8 y 2 para las bases en español y portugués, respectivamente). Por lo tanto, se incluyeron todos los autores cuyos documentos hubiesen sido citados al menos $1 \mathrm{vez}$, resultando en un total de 23 documentos en ambos idiomas.

De los documentos seleccionados en los tres idiomas se excluyeron aquellos que no contaban con versión digital y aquellos que carecían de resumen, introducción o conclusión, elementos necesarios para su registro, revisión y análisis. De forma tal que la base final quedó integrada por 32 documentos en inglés y 14 en español y portugués (ver anexo 1).

A continuación, estos documentos fueron sometidos a un análisis de contenido, método que procura generar conocimiento mediante el examen e identificación de patrones, temas y significados pertinentes al objetivo de la investigación. De acuerdo con Guest, Namey y Mitchell (2013), cabe distinguir dos técnicas de análisis de contenido: guiada por hipótesis o por contenidos. El presente estudio utiliza la segunda opción. De este modo, se distingue si el capitalismo académico es descrito como un fenómeno relativo a las dinámicas de economía política de los sistemas o como una transformación en la organización universitaria. Los resultados se muestran en la tabla 1 (para una versión detallada de esta tabla, véase anexo 2).

\section{Las dimensiones del capitalismo académico: una propuesta de análisis}

Como se observa en el cuadro anterior, en lo que respecta a la comprensión del fenómeno del capitalismo académico, es posible identificar dos vertientes claramente diferenciadas de análisis en la literatura especializada. Por una parte, una enfocada en el nivel macro de la economía política de los sistemas de educación superior que aborda temas relacionados con el reemplazo del Estado y las comunidades académicas por el mercado como principal mecanismo de coordinación (Slaughter y Leslie, 2001; Slaughter y Rhoades, 2004; Fernández, 2009), el aumento de proveedores privados (Münch, 2014; Kauppinen y Kaidesoja, 2014; Azevedo, 2015), la creciente relevancia del pago de aranceles (Ibarra, 2005; Johnson y Hirt, 2011; Metcalfe, 2016), la venta de productos y servicios de conocimiento distintos de la docencia en el financiamiento de la educación superior (Leslie, Rhoades y Oaxaca, 1999; Paasi, 2015; Fernández, 2011) y la mayor participación de estas instituciones en los mercados financieros mediante la compra y venta de sus activos (Kauppinen, 2012, 2013; Rhoades y TorresOlave, 2015). Por otra parte, la vertiente que subraya cómo el capitalismo académico transforma internamente a las organizaciones académicas apunta a sus consecuencias, que serían

TAbla 1. Foco de la literatura especializada en el capitalismo académico

Economía política Organizaciones

Literatura sobre capitalismo académico en castellano y portugués

Total
17

15

9

26

20
Total

32

$5 \quad 14$


principalmente la adopción de comportamientos y valores emprendedores (Renault, 2006; Mendoza, 2012; Fioreze, 2017) y una mayor influencia en las decisiones por parte del estrato de la alta administración, en desmedro de los cuerpos académicos (Fernández, Rodríguez y Rodríguez, 2010; Mars y Rhoades, 2012; Glenna et al., 2007).

La polisemia del concepto de capitalismo académico, que llevada al extremo puede generar confusión, puede reducirse si las diferentes definiciones presentes en la literatura se ponen en diálogo entre sí y con otras tradiciones de estudio de la educación superior. A continuación se realiza este ejercicio para el caso de la primera de las dos vertientes reseñadas, que aborda el estudio de los fenómenos de economía política de los sistemas de educación superior, los cuales proponemos clasificar en procesos de mercadización, privatización, mercantilización, comercialización y financiarización, con sus efectos organizacionales emergentes de universidades emprendedoras y de gerencialismo de la gestión. Distinguir entre estas diferentes dimensiones del capitalismo académico, como se verá más adelante, resulta imprescindible para su aplicación al estudio de casos nacionales.

\section{Capitalismo académico y economía política}

\section{Mercadización}

La mercadización (marketization) se refiere al rol activo que los mercados asumen en la coordinación y gobernanza de los sistemas de educación superior, dinámica descrita por primera vez por Clark (1984) de manera sistemática. Este autor identificó como mercados relevantes el de instituciones proveedoras; el de estudiantes; el de personal académico, directivo y gerencial; el de recursos; y el mercado de prestigios, de creciente importancia a nivel mundial hoy en día (Ordorika y Lloyd, 2015). Un mercado adicional, que ocupa un lugar central en la conducción y orientación de las instituciones formativas, es el mercado laboral de técnicos superiores y profesionales (Ferreyra et al., 2017). En términos generales, la mercadización consiste en una intensificación del papel que uno o más de estos mercados desempeñan en la educación superior, modificando el uso de los mecanismos de control y comando estatales y de autorregulación de las universidades (Teixeira et al., 2004). La literatura especializada muestra que se trata de un fenómeno global (Altbach, Reisberg y Rumbley, 2009), cuya intensidad varía entre sistemas nacionales (Wedlin, 2008).

\section{Privatización}

La privatización comprende dos aspectos. Por un lado, la privatización de la provisión, fenómeno que acompaña a la aparición de proveedores privados de educación superior, instituciones variadas que conforman un sector distintivo de oferta de educación terciaria, especialmente activo en las fases de fuerte expansión de la matrícula (Levy, 2013). Según las circunstancias de cada contexto, la matrícula privada puede ser marginal, mediana o mayoritaria; puede ser provista por instituciones con o sin fines de lucro; puede ser de nivel universitario o técnico-vocacional y gozar o no de apoyo directo del Estado. Igualmente, los estudiantes que cursan sus estudios en instituciones privadas pueden, o no, tener acceso a becas y/o créditos estudiantiles subsidiados con fondos fiscales. En América Latina, donde algo más de la mitad de la matrícula de educación superior se halla registrada en instituciones privadas (Brunner y Miranda, 2016), su variedad cubre todo ese espectro de posibilidades. En efecto, hay instituciones privadas con diferente nivel de prestigio, tamaño, misión, antigüedad, especialidad, composición de su cuerpo académico, perfil socioeconómico de sus alumnos, valor de los aranceles, solidez de su acreditación y su forma de gobierno. Por otro lado, la privatización del financiamiento de los sistemas e instituciones, consistente en el hecho de que una proporción creciente, en cualquier caso mayoritaria 
o significativa, de los recursos proviene de fuentes privadas, como son los hogares que pagan la educación superior de sus miembros, o los propios estudiantes que pagan directamente de su bolsillo o mediante préstamos públicos o comerciales, o de otras entidades privadas que concurren a financiar la educación superior. En términos empleados por Marginson (2018), se trata en este caso a la educación superior como bienes privados, en tanto que son producidos por el mercado y tratados como pertenecientes al sector no estatal. En América Latina hay una variedad de ejemplos del avance experimentado por el financiamiento privado de instituciones y sistemas de educación superior, así como de las ideologías y políticas que lo impulsan y sus efectos para estudiantes, organizaciones, sistemas, sociedades y Estados (Ferreyra et al., 2017).

\section{Mercantilización}

La mercantilización (commodification) de la provisión es un fenómeno que ocurre desde el momento en que el bien educación superior ingresa en la esfera de los intercambios de mercado y los estudiantes, o algunos de ellos, pagan un precio por los estudios, convirtiéndose, como suele decirse, en clientes (Giroux, 2014). De hecho, la mercantilización o comodificación característica del capitalismo académico contemporáneo es parte de una dinámica más amplia, abarcando el conocimiento avanzado en toda su extensión, tanto en las fases de producción como de transmisión, acumulación, transferencia y aplicación (Kauppinen, 2014). Max Weber habla de "mercabilidad" de los bienes (Weber, 2014: 209); esto es, la frecuencia con que estos acostumbran a ser objeto de cambio en el mercado. A lo largo de la historia, algunos bienes se han mantenido al margen de los mercados porque son considerados sagrados (Weber, 2014) o son tratados como bienes públicos (Marginson, 2018) o, por el motivo que sea, son declarados no comercializables (Samuelson, 1954). En la actualidad, la educación superior —que durante el siglo XIX y parte del siglo XX fue considerada predominantemente como un bien público, de provisión gratuita, financiado por el Estado (Marginson, 2011)_ está siendo incorporada, en un gran número de países, plena o parcialmente al mercado y circula allí como una mercancía bajo las reglas de la competencia (Naidoo y Jamieson, 2005).

\section{Comercialización}

En el marco más general de la comodificación del conocimiento, la mercantilización típica del capitalismo académico se acompaña de procesos de comercialización (commercialization), referidos a la incorporación de otras actividades universitarias —distintas de la educación formal conducente a grados y títulos académicos-, al mercado, extendiéndose a una amplia gama de servicios y productos que las instituciones proveen con el fin de generar ingresos (Slaughter y Leslie, 1997; Slaughter y Rhoades, 2004; Münch, 2014). Caben aquí, por ejemplo, trabajos de asesoría, consultoría, evaluación, test de diversa naturaleza, programas de capacitación y, sobre todo, la comercialización de productos generados por las labores de $\mathrm{I}+\mathrm{D}+\mathrm{i}$, realizadas junto con empresas y agencias de gobierno. Si bien los fenómenos de "triple hélice" (Ranga y Etzkowitz, 2013) aparecen en el centro de las dinámicas del capitalismo académico en los países desarrollados, particularmente en el caso de las grandes universidades de investigación de Estados Unidos (Cantwell, Taylor y Johnson, 2018), en América Latina su contribución a la generación de ingresos es menor, salvo si se contabilizan los fondos públicos para investigación, que en algunos casos son una fracción significativa del presupuesto anual.

\section{Financiarización}

La financiarización (financialization) abarca una amplia gama de actividades (Aalbers, 2019) que ponen en contacto directo a las instituciones de educación superior con los mercados financieros para obtener préstamos de largo 
plazo, invertir en instrumentos financieros, adquirir y vender instituciones o participación en ella, atraer inversiones directas del extranjero, desarrollar mercados secundarios para créditos estudiantiles y administrar portafolios de inversiones financieras (Jessop, 2017). Esto incide fuertemente en los modos de financiar la educación superior. Como se ha visto anteriormente, una proporción creciente de recursos proviene ahora de fuentes privadas bajo la forma de aranceles pagados por los estudiantes y sus familias, la venta de productos y servicios de conocimiento y una progresiva financiarización por medio de la presencia activa de las instituciones en el mercado financiero (Altbach, Reisberg y Rumbley, 2009; Hoyos, 2012; López Zárate, 2014).

\section{Capitalismo académico y cambio organizacional}

De manera simultánea, emerge una doble tendencia a nivel de las instituciones entendidas como organizaciones académicas. Por un lado, hacia la adopción de alguna modalidad de universidad emprendedora (entrepreneurial), con el fin de adaptarse a cambios en su entorno (Clark, 1984), obtener ingresos de diversas fuentes públicas y privadas y generar un excedente como base para mantener y desarrollar ventajas competitivas (Shattock, 2000). Por otro lado, la tendencia hacia el gerencialismo (managerialism) (Deem, 2017), manifestado en el reforzamiento de los gobiernos corporativos en su rama ejecutiva, la creación de un estamento de profesionales encargados de la gestión de las organizaciones académicas, el diseño e implementación de planes estratégicos, la preocupación por la efectividad y la eficiencia y la atención prestada a los usuarios (Stage y Aagaard, 2019). Este mismo gerencialismo (Sisto, 2017) adopta la forma de una nueva gestión pública en las universidades estatales (Broucker y De Wit, 2015), cuyas culturas organizacionales tradicionales se transforman también en un sentido emprendedor (Gibbs, Haskins y Robertson, 2009), fenómeno que se observa igualmente en países de América Latina (Brunner, 2014; Moreno Arellano, 2017).

\section{Un intento de superación del capitalismo académico: el caso chileno}

Esta sección pone a prueba el esquema conceptual de análisis del capitalismo académico descrito en la sección anterior, a propósito del estudio del caso chileno y su reciente reforma destinada a reducir sus niveles de privatismo.

En Chile, a partir de 1990, con la recuperación de la democracia después de 17 años de dictadura autoritaria, las políticas gubernamentales reconocen las bases de un régimen mixto de economía política de la educación superior nacional — con provisión y financiamiento estatal y privado-y utilizan una variada gama de instrumentos (Hall, 1993) para ampliar el acceso a la educación superior, asegurar la difusión de sus beneficios sociales, controlar y mejorar la calidad de la docencia y aumentar la pertinencia e impacto de la investigación, además de proporcionar a las instituciones un marco de reglas para su desarrollo y alineamiento con el bienestar general (Brunner, 2015). Sin embargo, el alto grado de privatismo alcanzado por la educación superior chilena fue ampliamente cuestionado en la esfera política, en particular por el movimiento estudiantil de los años 2011 y 2012 (Fleet, 2011). Como resultado, el Gobierno de Michel Bachelet (2014-2018), basado en una concepción que Marginson (2018) denomina "socialdemócrata de la educación superior" (bien no de mercado provisto por el sector estatal), buscó reducir el peso del factor y de las dinámicas privadas en la provisión y el financiamiento del sistema y, en cambio, fortalecer el compromiso público de las instituciones (González y Espinoza, 2017).

Desde el punto de vista de la literatura comparada, resulta de interés averiguar si este cambio de paradigma ambicionado por el Gobierno chileno resultó efectivamente en un nuevo 
rumbo para este sector, reduciendo el grado de privatismo en las dimensiones relevantes del capitalismo académico (Brunner, Ganga-Contreras y Rodríguez-Ponce, 2018). El análisis de este caso permite ilustrar las posibilidades del esquema conceptual provisto con anterioridad, tanto en las dimensiones de economía política (mercadización, privatización de la provisión y el financiamiento, mercantilización, comercialización, financiarización) como de las organizaciones académicas (universidad emprendedora y gerencialismo).

En tal sentido, el sistema chileno prereforma exhibía un grado avanzado de privatismo, de acuerdo con un arreglo de economía política que organizaba la provisión y el financiamiento de manera fuertemente dependiente de dinámicas privadas; con una coordinación sistémica basada en gran medida en la competencia y los mercados, y con tendencias manifiestas a favorecer organizaciones universitarias emprendedoras gestionadas gerencialmente (Brunner, 2014, 2017). En efecto, la mercadización se manifestaba de manera superlativa (Fernández y Bernasconi, 2012), afectando diferenciadamente a las instituciones, según si eran estatales, privadas dependientes de subsidios fiscales o sin aportes directos del Estado (Fernández, 2018). A su turno, en lo que respecta a la privatización, esta se expresaba de manera intensa y variada, existiendo oferentes privados en áreas técnicas, profesionales y académicas con calidades heterogéneas que, en conjunto, proveían un $85 \%$ de la matrícula del sistema (UIS, 2018). Asimismo, la mayor parte de los recursos provenía, tanto en las instituciones privadas como en las estatales, de fuentes privadas, originándose mediante el cobro de aranceles, los cuales, en el caso chileno, son considerados altos en comparación internacional (OECD, 2019: 317). En el año 2016, la proporción relativa del gasto privado en educación superior representó un 64\% del gasto total del sistema (OECD, 2019: 299).

Esto significa que la mercantilización del sistema chileno alcanzó un alto nivel, expresándose en el hecho de que, en todas las instituciones, incluidas las del Estado, los estudiantes debían pagar un arancel similar según la duración de los programas y el prestigio relativo de ellos. Sin embargo, a partir de 2015 se inicia la aplicación de una política de gratuidad en el acceso a los estudios superiores, en beneficio de los jóvenes de menores recursos, hasta cubrir a todos aquellos provenientes del $60 \%$ de los hogares de menores recursos de la población matriculados en instituciones elegibles (Delisle y Bernasconi, 2018). A continuación, en lo relativo a la comercialización de actividades de investigación e innovación, la mayoría de las instituciones otorgaba escasa atención a la venta de productos y servicios de este tipo, principalmente porque las actividades de investigación y desarrollo se hallaban concentradas en un número pequeño de instituciones y tenían orientación netamente académica. Con todo, varias habían comenzado a impulsar la comercialización de actividades docentes y de capacitación distintas de la enseñanza formal conducente a grados y títulos, consiguiendo por este medio recursos que en unos pocos casos representaban una fracción importante de sus presupuestos de ingreso (Bernasconi, 2011). Finalmente, la financiarización alcanzó un nivel elevado en el interior del sistema chileno de educación superior, básicamente por dos vías. Por un lado, el desarrollo de un esquema cada vez más amplio de crédito estudiantil administrado por la banca privada y acompañado por la creación de un mercado secundario de seguros de deuda (Student Loan Asset-Based Securities [SLABS]), un fenómeno relativamente nuevo en la práctica internacional comparada y ampliamente discutido en cuanto a sus efectos socioeconómicos y políticos (Jessop, 2017). Por otro lado, un agresivo esquema de inversiones, especialmente de instituciones privadas, las cuales, con respaldo del sistema financiero nacional y del régimen especial para la inversión directa extranjera, más la existencia de un subsector de instituciones con fines de lucro y la existencia de un mercado de transferencia del dominio o del control de las sociedades propietarias o controladoras 
de corporaciones universitarias y de educación superior (Bernasconi, 2013) desarrollaron una amplia infraestructura material y humana para dar cabida a una masa estudiantil que se expandió por casi cinco veces entre 1990 y 2018, así como a un volumen de actividad docente y de investigación vastamente más diferenciado y complejo, como se refleja ahora en el patrimonio neto total contenido en los estados financieros de instituciones de educación superior (Brunner y Labraña, 2018).

En lo que respecta al impacto del capitalismo académico a nivel de las organizaciones, diversos estudios apuntan hacia la difusión de comportamientos y valores emprendedores entre las universidades chilenas. En efecto, siguiendo las dimensiones identificadas por Clark (1984), se observa el desarrollo de una periferia de desarrollo organizacional expandida (Bernasconi, 2011; Brunner, 2008), una cultura emprendedora integrada (Guzmán-Valenzuela, Barnett y Labraña, 2019) y una progresiva diversificación de las fuentes de financiación (Pineda, 2015). Por otra parte, el gerencialismo adquirió también relevancia, manifestándose en la profesionalización de los estamentos directivos y su centralidad en la conducción de las universidades (Pedraja-Rejas y Rodríguez-Ponce, 2015; Rodríguez-Ponce et al., 2017).

La reforma de la educación superior chilena impulsada entre 2014 y 2018 buscó generar una transformación en todas estas dimensiones. Primero, impulsó una desmercadización parcial de la coordinación del sistema mediante la introducción de una nueva Ley de Educación Superior (Ley 21091, de 9 de mayo de 2018). Esta establece un conjunto de regulaciones, obligaciones, requisitos y exigencias para enmarcar el funcionamiento de los mercados y el comportamiento de las instituciones (León, 2018). Si bien la mayoría se encuentra en proceso de implementarse, durante su discusión parlamentaria se manifestaron reparos a su carácter restrictivo para la autonomía y el futuro dinamismo del sistema (Brunner, 2017).
Segundo, puso en marcha un proceso de desprivatización de la provisión, intentando incrementar la provisión estatal mediante la creación de dos nuevas universidades regionales y de quince centros de formación técnica. Este esfuerzo tuvo un efecto casi imperceptible; sin embargo, la participación de la matrícula estatal se mantuvo en un 16\% (SIES, 2018).

Tercero, en la misma dirección, la reforma intentó desprivatizar el financiamiento de la educación superior, corrigiendo al efecto - especialmente mediante la política de acceso gratuito a la educación superior- el desequilibrio entre bajo gasto fiscal y alto gasto privado. De hecho, el primero pasó de menos de un tercio del gasto inicial total en el sector en el año 2005 a un 46\% en 2016 (OECD, 2019: 300; Brunner y Labraña, 2018). Del total de recursos fiscales asignados al sistema, en 2017 se asignó un $73 \%$ a estudiantes: $34 \%$ en concepto de subsidio de gratuidad y $39 \%$ en concepto de créditos y becas. En consecuencia, a pesar de los esfuerzos de transformación, la mayor parte del gasto fiscal destinado a la educación superior continúa asignándose como un subsidio a la demanda. Por su lado, el subsidio a la oferta (i.e., instituciones e investigación académica) se distribuye en su mayoría mediante un aporte fiscal directo (no condicionado) que beneficia a las instituciones estatales y privadas dependientes. Los restantes recursos asignados por el Estado son distribuidos mediante contratos de desempeño, fondos concursables y otros dispositivos que vinculan recursos a desempeños y resultados, beneficiando igualmente a las instituciones universitarias mencionadas (CGR, 2018b).

Cuarto, en consecuencia, la reforma promovió efectivamente la desmercantilización al adoptar una política de gratuidad que, comenzando con los jóvenes de menores recursos, esperaba conseguir sustraer por completo la provisión de la educación superior del mercado a medio o largo plazo (León, 2018), reconociéndola como un derecho social según la clásica definición de T. S. Marshall y como un bien público 
social-democrático según la denominación de Marginson (2018). En adelante este derecho sería garantizado y solventado por el Estado, sin costo para los ciudadanos beneficiados, siendo financiado por recursos de la renta nacional (Delisle y Bernasconi, 2018). En la práctica, la gratuidad implementada desde 2017 favorece a los estudiantes del $60 \%$ de la población de menores recursos, esto es, a alrededor de un cuarto de la matrícula total (CGR, 2018).

Quinto, la reforma empujó también, al menos retóricamente, a una descomercialización de la academia, buscando reducir las actividades orientadas directamente a generar ingresos en el mercado. Con todo, en la medida en que el diseño de la gratuidad limita la libertad de las instituciones para incrementar el número de vacantes o aumentar el valor de los aranceles, provoca, como reacción, una mayor competencia por fondos públicos y privados de investigación y una mayor presión para extender innovadoramente la comercialización del conocimiento hacia otras esferas (Brunner y Labraña, 2018). La información estadística disponible indica que en el año 2017, un 66\% del ingreso total de las universidades provino del cobro de aranceles, en las universidades estatales correspondió a un $53,9 \%$, en las universidades privadas dependientes a un $56,1 \%$ y en las universidades privadas independientes a un $89,4 \%$. A su turno, los ingresos por prestación de servicios, venta de cursos y extensión y aquellos calificados como "otros ingresos" generaron un 12,6\%, un $21,7 \%$ y un $8,7 \%$, respectivamente, en aquellas tres categorías institucionales. Por fin, los fondos públicos basales y concursables representaron ese año un 33,2\%, un 21,5\% y un $0,4 \%$, respectivamente. Las donaciones, cuya cuantía es marginal — salvo en el caso de unas pocas universidades privadas-, aportaron el resto de los ingresos (CGR, 2018).

Sexto, las dos últimas Administraciones de gobierno (2014-2018 y 2018-2022) han buscado además una relativa desfinanciarización del sistema, sustituyendo el actual esquema de crédito estudiantil con aval del Estado e intermediación de la banca — cuya operación ha sido sometida a variadas críticas (Espinoza y González, 2016), pero también a evaluaciones positivas (Williamson et al., 2019) — por un esquema de crédito contingente al ingreso administrado por una agencia pública independiente (Gobierno de Chile, 2018). El proyecto de ley que materializa este cambio se encuentra actualmente en trámite en el Parlamento y ha recibido críticas de representantes del sector privado por mantener la fijación del precio de los aranceles y del sector de instituciones estatales por mantener la competencia como medio para la obtención de recursos y un esquema de préstamos que prolonga la vida de un paradigma neoliberal de política sectorial (Brunner, 2015).

Si se aceptan las anteriores consideraciones relacionadas con la influencia del capitalismo académico a nivel de economía política del sistema chileno de educación superior, entonces puede colegirse fácilmente que las políticas de reversión del privatismo adoptadas durante el último cuatrienio (2014-2018) no lograron el cambio de paradigma perseguido. En efecto, la economía política del sistema permanece incólume; la mercadización y la provisión privada se sujetan a reglas y regulaciones adicionales; se reduce en alguna medida la mercantilización en favor de estudiantes provenientes de familias de menores ingresos, modificándose de paso el perfil del endeudamiento estudiantil; la comercialización podría estar aumentando y la financiarización sigue adelante con la expectativa de que próximamente podría disminuir el papel de la banca privada en la administración de la deuda del crédito estudiantil. Dentro de este cuadro, cabe concluir además que los efectos de empresarialización de las instituciones universitarias y su gestión de tipo gerencial no deben haber disminuido significativamente tampoco, pudiendo incluso haberse exacerbado en algunos aspectos debido a la mayor presión evaluativa del Estado y al inicio de un estancamiento relativo del crecimiento económico del país. 
En suma, la incidencia del capitalismo académico en el sistema no parece haberse alterado significativamente, aunque su funcionamiento pudiese haberse mitigado en algunos aspectos, al mismo tiempo que su legitimidad podría a la larga salir fortalecida al expandirse el control público sobre el sistema.

Así pues, el examen sugiere que las políticas y medidas dirigidas a disminuir el privatismo en la educación superior chilena no han logrado su objetivo ni han superado las dinámicas de competencia por recursos propias del capitalismo académico. Por el contrario, bien podría terminar incrementándose el impacto de las tendencias de mercadización, privatización, mercantilización, comercialización y financiarización que aquellas políticas intentaron originalmente controlar. En consecuencia, cabe esperar que también las tendencias hacia la empresarialización y el gerencialismo continúen vigentes. Más aún, debido a que las universidades se han visto —o podrán verse próximamente - afectadas negativamente en sus ingresos por una política de gratuidad mal diseñada e implementada (Delisle y Bernasconi, 2018). Es posible que experimenten un aumento de la presión por volverse entidades emprendedoras y deban refinar y profundizar aún más sus capacidades de gestión para posicionarse de manera competitiva en los mercados locales y regionales de la educación superior.

TABla 2. Dinámicas de privatismo y políticas que apuntan a desprivatizar la ES: el caso de Chile (2014-2018)

Dinámicas que conforman el privatismo chileno

Mercadización: la gobernanza y coordinación del sistema de educación superior recurre ampliamente a los mercados y a mecanismos de tipo mercado

Privatización: provisión privada dominante

Privatización: los recursos que financian el sistema provienen fundamentalmente de fuentes privadas

Mercantilización: el bien educación superior es provisto en el mercado por un precio, tanto por instituciones estatales como privadas

Comercialización: venta de bienes y servicios de conocimiento diferentes de programas y cursos conducentes a un grado o título

Financiarización: participación de las instituciones de educación superior, para distintos fines y con diferentes medios, en los circuitos de la intermediación financiera

Efecto de dinámicas anteriores: empresarialización de instituciones y énfasis en el gerencialismo dentro de estas
Políticas destinadas a revertir el privatismo

Desmercadización para ampliar y diversificar la regulación de los mercados: fijación de aranceles, determinación de vacantes, mayores requisitos para el acceso a los mercados y, en general, un mayor peso en la conducción, regulación y evaluación estatales

Desprivatización para ampliar la oferta en instituciones estatales y eventualmente reducir la matrícula privada

El gasto del Estado aumenta su importancia dentro del total de recursos gastados en la educación superior, sustituyendo parcialmente recursos de origen privado

Desmercantilización para asegurar el acceso gratuito a la educación superior a un grupo de estudiantes y aumentar el subsidio fiscal para el crédito estudiantil, aligerando el endeudamiento de los jóvenes

Descomercialización al preverse una disminución de la necesidad de generar ingresos propios, expectativa que se frustra en la práctica al estancarse el gasto total en educación superior y, por ende, aumentar la presión competitiva por recursos

Desfinanciarización para el fin propuesto del mercado secundario de crédito estudiantil y otras medidas que limitan la necesidad o la posibilidad de las instituciones de recurrir al sistema financiero

Efecto buscado como una consecuencia de políticas anteriores de reducción del privatismo: limitar la empresarialización y el gerencialismo de las organizaciones académicas y, en lo posible, revertirlos 
La tabla 2 resume las diferentes dimensiones del capitalismo académico chileno antes y después de las políticas públicas orientadas a su superación.

\section{Conclusiones}

De acuerdo con una revisión de la literatura especializada, este artículo plantea que existen dos modos de comprender el capitalismo académico: el primero, a nivel del sistema, centrado en los cambios de la economía política de la educación superior en el ámbito nacional y global. El segundo, enfocado en sus efectos a nivel micro dentro de las organizaciones académicas. A continuación, siguiendo esta distinción y en diálogo con otras tradiciones de estudio de la educación superior, sugerimos un concepto integrado de capitalismo académico como un proceso de transformación en las dimensiones de mercadización, privatización de la provisión y del financiamiento, mercantilización, comercialización y financiarización (nivel de economía política), la cual da lugar a cambios de comportamiento y cultura organizacionales en dirección a valores emprendedores y un mayor gerencialismo de la gestión (nivel de las instituciones). Por último, probamos este marco de análisis examinando el caso chileno antes y después de las reformas destinadas a reducir la incidencia del capitalismo académico en su sistema nacional de educación superior.

El marco conceptual propuesto abre diversas perspectivas para futuros estudios. En primer lugar, una especificación de las dimensiones que caracterizan al capitalismo académico, tanto a nivel de economía política como en el plano organizacional, abre las puertas hacia análisis contextualizados de diferentes sistemas nacionales de educación superior. Atentos a las diferencias locales del capitalismo académico, el enfoque propuesto se aleja de las comprensiones homogéneas de este fenómeno y de la noción de un capitalismo académico único. Permitiría avanzar, en cambio, en la identificación de una variedad de capitalismos académicos y, a nivel organizacional, en la identificación de sus efectos según los tipos de instituciones de educación superior (Brunner et al., 2019; Mendoza, 2012).

El hecho de que el marco analítico propuesto facilite el diálogo entre la teoría del capitalismo académico y otros enfoques teóricos en el campo de la educación superior — como la sociología de estos sistemas, el enfoque organizacional, la economía política y las teorías de la gobernanza y la gestión- permite vincular el desarrollo de aquella teoría con perspectivas que están en proceso de construcción y consolidación del campo de estudio de la educación superior (De Oliveira Andreoti et al., 2016; Huisman y Tight, 2016).

Finalmente, en el ámbito de las políticas públicas, el marco señalado favorecería análisis más precisos de las complejas dinámicas que constituyen al capitalismo académico como fenómeno a nivel de sistemas nacionales y sus efectos a nivel de las instituciones. En efecto, distinguir cómo las reformas afectan el grado de mercadización, privatización, mercantilización, comercialización y financiarización y, con ello, la empresarialización y el gerencialismo de las instituciones permitiría dar cuenta de cómo las reformas pueden producir consecuencias no anticipadas (De Zwart, 2015), de manera que en vez de reducir los grados de privatismo en la educación superior pueden terminar manteniéndolo e incluso reforzándolo a veces en algunos aspectos, efectos que contradicen las intenciones originales de sus promotores. Solo últimamente ha comenzado a abordarse este tipo de análisis y sus consecuencias en algunos países: según lo esperado en el caso de Polonia (Kwiek, 2017) y, en cambio, con efectos ambiguos y no anticipados en el caso de Chile (Brunner, Ganga-Contreras y Rodríguez-Ponce, 2018), al igual que sus límites para alterar la lógica institucional del capitalismo académico (Fini y Toschi, 2016). 


\section{Referencias bibliográficas}

Aalbers, M. B. (2019). Financialization. En D. Richardson, N. Castree, M. F. Goodchild, A. L. Kobayashi y R. Marston (eds.), The international encyclopedia of geography: people, the Earth, environment, and technology. Oxford: Wiley.

Altbach, P. G., Reisberg, L. y Rumbley, L. E. (2009). Trends in global higher education: tracking an academic revolution. París: UNESCO.

Azevedo, M. (2015). Transnacionalização e mercadorização da educação superior: Examinando alguns efeitos colaterais do capitalismo acadêmico (sem riscos) no Brasil - A expansão privadomercantil. Revista Internacional de Educação Superior, 1(1), 86-102.

Bastedo, M. N. (ed.) (2012). The organization of higher education: managing colleges for a new era. Baltimore: The John Hopkins University Press.

Bernasconi, A. (2011). Public and private pathways to world-class research universities: the case of Chile. En G. Altbach y J. Salmi (eds.), The road to academic excellence: the making of world-class universities (pp. 229-260). Washington, D. C.: World Bank.

Bernasconi, A. (2013). Prohibición del lucro en las universidades: alcance y consecuencias. Centro de Políticas Públicas UC: temas de la agenda pública, 8(62). Recuperado de https://politicaspublicas.uc.cl/wp-content/ uploads/2015/02/serie-no-62-prohibicion-del-lucro-en-las-universidades-alcance-y-consecuencias.pdf

Broucker, B. y De Wit, K. (2015). New public management in higher education. En J. Huisman, H. de Boer, D. D. Dill y M. Souto-Otero (eds.), Handbook of higher education policy (pp. 57-74). Dordrecht: Springer.

Brunner, J. J. (2008). El sistema de educación superior en Chile: un enfoque de economía política comparada. Avaliação: Revista da Avaliação da Educação Superior (Campinas), 13(2), 451-486. doi: https://doi.org/10.1590/S1414-40772008000200010

Brunner, J. J. (2014). La idea de la universidad pública en América Latina: narraciones en escenarios divergentes. Educación XXI, 17(2), 17-34. doi: https://doi.org/10.5944/educxx1.17.2.11477

Brunner, J. J. (2015). Medio siglo de transformaciones de la educación superior chilena: un estado del arte. En A. Bernasconi (ed.), La educación superior de Chile: transformación, desarrollo y crisis (pp. 21-107). Santiago, Chile: Universidad Católica de Chile.

Brunner, J. J. (2017). La economía política del capitalismo académico: el caso de Chile. En H. Lavados y A. M. Durán (eds.), Desafíos para el desarrollo universitario en Chile (pp. 159-231). Valencia: Tirant Humanidades.

Brunner, J. J., Ganga-Contreras, F. y Rodríguez-Ponce, E. (2018). Gobernanza del capitalismo académico: aproximaciones desde Chile. Revista Venezolana de Gerencia, 23(1), 11-35.

Brunner, J. J., Labraña, J., Ganga, F. y Rodríguez-Ponce, E. (2019). Circulación y recepción de la teoría del "academic capitalism" en América Latina. Archivos Analíticos de Políticas Educativas, 27(79).

Brunner, J. J. y Miranda, D. A. (eds.) (2016). Educación superior en Iberoamérica: informe 2016. Santiago, Chile: Centro Interuniversitario de Desarrollo.

Brunner, J. J. y Labraña, J. (2018). Financiamiento de la educación superior, gratuidad y proyecto de nuevo crédito estudiantil. Centro de Políticas Públicas: Debates de Política Pública, 31. Recuperado de https://www.cepchile.cl/cep/site/artic/20181115/asocfile/20181115124945/dpp_031_noviembre2018_jjbrunner_jlabrana.pdf

Cantwell, B. y Kauppinen, I. (eds.) (2014). Academic capitalism in the age of globalization. Baltimore: Johns Hopkins University Press.

Cantwell, B., Taylor, B. J. y Johnson, N. M. (2018). Ordering the global field of academic science: money, mission, and position. Studies in Higher Education, 45(1), 1-16. doi: https://doi.org/10.10 80/03075079.2018.1506916 
Capitalismo académico: distinciones conceptuales y procesos contradictorios a propósito del caso chileno

CGR (Contraloría General de la República) (2018). Financiamiento fiscal a la educación superior 2017. Santiago, Chile: Contraloría General de la República.

Clark B. R. (ed.) (1984). Perspectives in higher education. Berkeley: University of California Press.

De Oliveira Andreotti, V., Stein, S., Pashby, K. y Nicolson, M. (2016). Social cartographies as performative devices in research on higher education. Higher Education Research \& Development, 35(1), 84-99. doi: 10.1080/07294360.2015.1125857

De Zwart, F. (2015). Unintended but not unanticipated consequences. Theory and Society, 44(3), 283-297. doi: 10.1007/s11186-015-9247-6

Deem, R. (2017). New managerialism in higher education. En J. Shin J. y P. Teixeira (eds.), Encyclopedia of international higher education systems and institutions. Dordrecht: Springer.

Delisle, J. y Bernasconi, A. (2018). Lessons from Chile's transition to free college. Evidence Speaks Reports, 2(43).

Espinoza, O. y González, L. E. (2016). La educación superior en Chile y la compleja transición desde el régimen de autofinanciamiento hacia el régimen de gratuidad. Revista Latinoamericana de Educación Comparada, 7(10), 35-51.

Fernández, E. (2009). El sistema-mundo del capitalismo académico: procesos de consolidación de la universidad emprendedora. Archivos Analíticos de Políticas Educativas, 17(21), 1-43.

Fernández, E. (2018). Política pública, mercado y diversidad institucional: las complejidades de clasificar instituciones de educación superior. El ejemplo chileno. INNOVAR. Revista de Ciencias Administrativas y Sociales, 28(67), 147-158. doi: https://doi.org/10.15446/innovar.v28n67.68620

Fernández, E. y Bernasconi, A. (2012). Elementos conceptuales para el análisis organizacional de universidades en contextos de mercado. INNOVAR. Revista de Ciencias Administrativas y Sociales, 22(46).

Fernández, E., Rodríguez, H. y Rodríguez, M. (2010). La formación inicial de profesionales de la educación: un análisis crítico de los nuevos planes de estudio en el contexto del "capitalismo académico". Revista Interuniversitaria de Formación del Profesorado, 24(2), 151-174.

Fernández, R. (2011). Capitalismo académico en la educación superior chilena ¿Modelo exitoso para el desarrollo de la ciencia? Análisis de las tendencias en la productividad científica en investigación durante la última década. Science for Innovation, 1(1), 36-45.

Ferreyra, M. M., Avitabile, C., Botero Álvares, J., Haimovich, F. y Urzúa, S. (2017). At a crossroads: higher education in Latin America and the Caribbean. Washington, D. C.: World Bank Publications.

Fini, R. y Toschi, L. (2016). Academic logic and corporate entrepreneurial intentions: a study of the interaction between cognitive and institutional factors in new firms. International Small Business Journal, 34(5), 637-659. doi: https://doi.org/10.1177/0266242615575760

Fioreze, C. (2017). O modelo comunitário de universidade e o tensionamento público-privado: entre o capitalismo acadêmico e o compromisso social. Porto Alegre, Brasil: Universidade Federal do Rio Grande do Sul.

Fleet, N. (2011). Movimiento estudiantil y transformaciones sociales en Chile: una perspectiva sociológica. Polis, Revista Latinoamericana, 10(30), 99-116. doi: https://doi.org/10.4067/S071865682011000300005

Gibb, A., Haskins, G. y Robertson, I. (2009). Leading entrepreneurial university: meeting the entrepreneurial development needs of higher education institutions. Londres: National Council for Graduate Entrepreneurship.

Giroux, H. A. (2014). Neoliberalism's war on higher education. Chicago, Illinois: Haymarket Books.

Glenna, L., Lacy, W., Welsh, R. y Biscotti, D. (2007). University administrators, agricultural biotechnology, and academic capitalism: defining the public good to promote university-industry relationships. The Sociological Quarterly, 48(1), 141-163. 
Gobierno de Chile (2018). Mensaje n. ${ }^{\circ}$ 043-366. Proyecto de ley que crea un nuevo sistema de financiamiento solidario para estudiantes de la educación superior. Santiago, Chile: Gobierno de Chile.

González, L. E. y Espinoza, O. (2017). Educación superior bajo el segundo Gobierno de Michelle Bachelet: una mirada evaluativa. En Bachelet II: el difícil camino hacia un Estado democrático social de derechos (pp. 167-184). Santiago, Chile: Barómetro de Política y Equidad.

Guest, G., Namey, E. E. y Mitchell, M. (2013). Collecting qualitative data: a field manual for applied research. Los Angeles, Londres, Nueva Delhi, Singapur y Washington D. C.: SAGE Publications.

Guzmán-Valenzuela, C., Barnett, R. y Labraña, J. (2019). Consensus and dissensus: changing perceptions of the public dimension of universities in a marketised environment. Journal of Higher Education Policy and Management.

Hall, P. (1993). Policy paradigms, social learning, and the state: the case of economic policy making in Britain. Comparative Politics, 23(4), 275-296.

Harzing, A. W. y Van der Wal, R. (2008). Google Scholar as a new source for citation analysis. Ethics in Science and Environmental Politics, 8(1), 61-73.

Hoyos, G. (2012). Democratizar la democracia: Latinoamérica y su universidad. Bordón. Revista de Pedagogía, 64(3), 117-131.

Huisman, J. y Tight, M. (eds.) (2016). Theory and method in higher education. Bingley, Reino Unido: Emerald.

Ibarra, E. (2005). "Capitalismo académico" en los márgenes: notas sobre la naturaleza de las transformaciones recientes de las universidades mexicanas. Texto presentado en el seminario permanente: Internacionalización de la Educación Superior: El Capitalismo Académico, Implicaciones para los Países en Desarrollo. Centro de Estudios sobre la Universidad, México, 21 y 22 de abril de 2005.

Jessop, B. (2017). Varieties of academic capitalism and entrepreneurial universities. Higher Education, 73(6), 853-870. doi: https://doi.org/10.1007/s10734-017-0120-6

Johnson, A. T. y Hirt, J. B. (2011). Reshaping academic capitalism to meet development priorities: the case of public universities in Kenya. Higher Education, 61. doi: 10.1007/s10734-010-9342-6

Kauppinen, I. (2012). Towards transnational academic capitalism. Higher Education, 64(4), 543-556. doi: https://doi.org/10.1007/s10734-012-9511-x

Kauppinen, I. (2013). Academic capitalism and the informational fraction of the transnational capitalist class. Globalisation, Societies and Education, 11(1). doi: https://doi.org/10.1080/1476772 4.2012 .678763

Kauppinen, I. (2014). Different meanings of knowledge as commodity in the context of higher education. Critical Sociology, 40(3), 393-409. doi: https://doi.org/10.1177/0896920512471218

Kauppinen, I. y Kaidesoja, T. (2014). A shift towards academic capitalism in Finland. Higher Education Policy, 27(1), 23-41. doi: 10.1057/hep.2013.11

Kwiek, M. (2017). De-privatization in higher education: a conceptual approach. Higher Education, 74(2), 259-281. doi: https://doi.org/10.1007/s10734-016-0047-3

León, J. J. (2018). Derecho y política de la educación superior chilena: evolución, crisis y reforma. Leiden: Universidad de Leiden.

Leslie, L., Rhoades, G. y Oaxaca, R. (1999). Technology transfer and academic capitalism. En A. H. Teich, S. D. Nelsson, C. McEnaney y S. J. Lita (eds.), AAAS Science and Technology Policy Yearbook, 2001. Nueva York.

Levy, D. C. (2013). The decline of private higher education. Higher Education Policy, 26, 25-42. doi: https://doi.org/10.1057/hep.2012.26

Ley 21091 (2018). Sobre educación superior. Santiago, Chile: Ministerio de Educación. 
Capitalismo académico: distinciones conceptuales y procesos contradictorios a propósito del caso chileno

López Zárate, R. (2014). La interacción gobierno-universidades: una relación compleja. Bordón. Revista de Pedagogía, 66(1), 75-88. doi: https://doi.org/10.13042/Bordon.2014.66105

Marginson, S. (2011). Higher education and public good. Higher Education Quarterly, 65(4), 411433. doi: 10.1111/j.1468-2273.2011.00496.x

Marginson, S. (2018). Public/private in higher education: a synthesis of economic and political approaches. Studies in Higher Education, 43(2), 322-337. doi: https://doi.org/10.1080/03075079.2 016.1168797

Mars, M. M. y Rhoades, G. (2012). Socially oriented student entrepreneurship: a study of student change agency in the academic capitalism context. The Journal of Higher Education, 83(3), 435459. doi: https://doi.org/10.1080/00221546.2012.11777251

Martínez, M. A., Herrera, M., López-Gijón, J. y Herrera-Viedma, E. (2014). H-Classics: characterizing the concept of citation classics through H-index. Scientometrics, 98(3), 1971-1983.

Mendoza, P. (2012). The role of context in academic capitalism: the industry-friendly department case. The Journal of Higher Education, 38(1), 26-48. doi: https://doi.org/10.1080/00221546.2012.11777233

Metcalfe, A. S. (2016). Revisiting academic capitalism in Canada: no longer the exception. The Journal of Higher Education, 81 (4), 489-514. doi: https://doi.org/10.1080/00221546.2010.11779062

Moreno Arellano, C. I. (2017). Las reformas en la educación superior pública en México: rupturas y continuidades. Revista de la Educación Superior, 46(182), 27-44. doi: https://doi.org/10.1016/j. resu.2017.03.001

Münch, R. (2014). Academic capitalism: universities in the global struggle for excellence. Nueva York y Londres: Taylor \& Francis.

Naidoo, R. y Jamieson, I. (2005). Knowledge in the marketplace: the global commodification of teaching and learning in higher education. En P. Ninnes y M. Hellsten (eds.), Internationalizing higher education: critical explorations of pedagogy and policy (pp. 37-52). Hong Kong: Springer.

OECD (Organisation for Economic Co-operation and Development) (2019). Education at a glance 2019: OECD indicators. París: OECD Publishing.

Ordorika, I. y Lloyd, M. (2015). International rankings and the contest for university hegemony. Journal of Education Policy, 30(3), 385-405. doi: https://doi.org/10.1080/02680939.2014.979247

Paasi, A. (2015). Academic capitalism and the geopolitics of knowledge. En J. Agnew, V. Mamadouh, A. J. Secor y J. Sharp (eds.), The Wiley Blackwell Companion to political geography (pp. 509-523). Nueva Jersey, Estados Unidos: Wiley-Blackwell.

Pedraja-Rejas, L. y Rodríguez-Ponce, E. (2015). Dirección estratégica y gestión en instituciones de educación superior. En A. Bernasconi (ed.), La educación superior de Chile: transformación, desarrollo y crisis (pp. 475-515). Santiago, Chile: Universidad Católica de Chile.

Pineda, P. (2015). The entrepreneurial research university in Latin America: global and local models in Chile and Colombia, 1950-2015. Nueva York: Palgrave Macmillan.

Ranga, M. y Etzkowitz, H. (2013). Triple helix systems: an analytical framework for innovation policy and practice in the knowledge society. Industry and Higher Education, 27, 237-262. doi: 10.5367/ihe.2013.0165

Renault, C. S. (2006). Academic capitalism and university incentives for faculty entrepreneurship. The Journal of Technology Transfer, 31 (2), 227-239. doi: https://doi.org/10.1007/s10961-005-6108-x

Rhoades, G. y Torres-Olave, B. (2015). Academic capitalism and (secondary) academic labor markets: negotiating a new academy and research agenda. En M. B. Paulsen (ed.), Higher education: handbook of theory and research: volume 30 (pp. 383-430). Nueva York, Dordrecht y Londres: Springer.

Rodríguez-Ponce, E., Pedraja-Rejas, L., Delgado-Almonte, M. y Ganga-Contreras, F. (2017). La relación entre la gestión financiera y la calidad en las instituciones de educación superior. Interciencia, 42(2), 119-126. 
Samuelson, P. (1954). The pure theory of public expenditure. Review of Economics and Statistics, 36(4), 387-389. doi: https://doi.org/10.2307/1925895

Shattock, M. (2000). Strategic management in European universities in an age of increasing institutional self-reliance. Tertiary Education and Management, 6(2), 93-104. doi: https://doi.org/10.108 0/13583883.2000.9967015

SIES (Servicio de Información de la Educación Superior) (2018). Informe matrícula 2018 en educación superior en Chile. Santiago, Chile: Ministerio de Educación.

Sisto, V. (2007). Managerialismo y trivialización de la universidad. Nómadas, 27(27), 8-21.

Slaughter, S. y Leslie, L. L. (1997). Academic capitalism: politics, policies, and the entrepreneurial university. Baltimore: Johns Hopkins University Press.

Slaughter, S. y Leslie, L. L. (2001). Expanding and elaborating the concept of academic capitalism. Organization, 8(2), 154-161.

Slaughter, S. y Rhoades, G. (2004). Academic capitalism and the new economy. Markets, state and higher education. Baltimore y Londres: Johns Hopkins University Press.

Stage, K. y Aagaard, K. (2019). Danish universities under transformation: developments in staff categories as indicator of organizational change. Higher Education, 78(4), 1-24. doi: https://doi. org/10.1007/s10734-019-00362-y

Teixeira, P., Jongbloed, B., Dill, D. y Amaral, A. (eds.) (2004). Markets in higher education. Rhetoric or reality? Dordrecht: Kluwer.

Tight, M. (2019). Higher education research. The developing field. Londres: Bloomsbury Academic. UIS (UNESCO Institute for Statistics) (2018). Global database education. París: UNESCO.

Weber, M. (2014). Economía y sociedad. Nueva edición revisada, comentada y anotada por Francisco Gil Villegas. México, D. F: Fondo de Cultura Económica.

Wedlin, L. (2008). University marketization: the process and its limits. En L. Engwall y D. Weaire (eds.), The university in the market (pp. 143-153). Londres: Portland Press.

Williamson, C., Kutcher, M., Riquelme, C. y Santelices, M. V. (2019). Tuition fees, student financial aid and higher education enrollment: the case of Chile. En M. V. Santelices, C. Horn y X. Catalán (eds.), The quest for equity in Chile's higher education: decades of continued efforts (pp. 59-78). Maryland: Lexington Books.

\section{Anexo 1}

Referencias de documentos utilizados en inglés

\begin{tabular}{lll} 
Autor/es & $\begin{array}{c}\text { Año de } \\
\text { publicación }\end{array}$ & Título \\
\hline Choi, P. K. & 2010 & $\begin{array}{l}\text { "Weep for Chinese university": a case study of English } \\
\text { hegemony and academic capitalism in higher education } \\
\text { in Hong Kong }\end{array}$ \\
\hline Deem, R. & 2001 & $\begin{array}{l}\text { Globalisation, new managerialism, academic capitalism and } \\
\text { entrepreneurialism in universities: is the local dimension still } \\
\text { important? }\end{array}$ \\
\hline Enders, J. & 2001 & $\begin{array}{l}\text { Between state control and academic capitalism: a comparative } \\
\text { perspective on academic staff in Europe }\end{array}$ \\
\hline $\begin{array}{l}\text { Glenna, L., Lacy, W., Welsh, R. } \\
\text { y Biscotti, D. }\end{array}$ & 2007 & $\begin{array}{l}\text { University administrators, agricultural biotechnology, and } \\
\text { academic capitalism: defining the public good to promote } \\
\text { university-industry relationships }\end{array}$ \\
\hline
\end{tabular}


Año de publicación

Gonzales, L. D., Martinez, E. y Ordu, C.

Hanley, L.

Hoffman, S. G.

Johnson, A. T. y Hirt, J. B.

Kauppinen, I.

Kauppinen, I. y Kaidesoja, T. Leslie, L., Rhoades, G. y Oaxaca, R.

Mars, M. M. y Rhoades, G.

2012

Mendoza, P.

2016

2016

Mendoza, P. y Berger, J.

Mendoza, P., Kuntz, A. M. y Berger, J. B.

Metcalfe, A. S.

2016

Münch, R.

Paasi, A.
Renault, C. S.
Rhoades, G.
Rhoades, G. y Slaughter, S.
Rhoades, G. y Torres-Olave, B.
Slaughter, S. y Leslie, L. L.
Slaughter, S. y Rhoades, G.
Welsh, R., Glenna, L., Lacy, W.
y Biscotti, D.

Ylijoki, O. H.

, A. yerger,

2008

2016

2014

2013

2015

2016

2006

2005

1997

2004

2015

2001

2004

2008

2003
Título

Exploring faculty experiences in a striving university through the lens of academic capitalism Academic capitalism in the new university

The new tools of the science trade: contested knowledge production and the conceptual vocabularies of academic capitalism

Reshaping academic capitalism to meet development priorities: the case of public universities in Kenya Towards transnational academic capitalism

Academic capitalism and the informational fraction of the transnational capitalist class

Towards a theory of transnational academic capitalism

A shift towards academic capitalism in Finland

Technology transfer and academic capitalism

Socially oriented student entrepreneurship: a study of student change agency in the academic capitalism context

The role of context in academic capitalism: the industryfriendly department case

Academic capitalism and doctoral student socialization: a case study

Academic capitalism and academic culture: a case study

Bourdieu and academic capitalism: faculty "habitus" in materials science and engineering

Revisiting academic capitalism in Canada: no longer the exception

Academic capitalism: universities in the global struggle for excellence

Fennia: positioning a peripheral but international journal under the condition of "academic capitalism"

Academic capitalism and the geopolitics of knowledge

Globalisation, academic capitalism, and the uneven geographies of international journal publishing spaces

Academic capitalism and university incentives for faculty entrepreneurship Capitalism, academic style, and shared governance Academic capitalism, managed professionals, and supply-side higher education Academic capitalism in the new economy: challenges and choices Academic capitalism and (secondary) academic labor markets: negotiating a new academy and research agenda Expanding and elaborating the concept of academic capitalism

Academic capitalism and the new economy: markets, state, and higher education

Close enough but not too far: assessing the effects of university-industry research relationships and the rise of academic capitalism

Entangled in academic capitalism? A case-study on changing ideals and practices of university research 


\section{Referencias de documentos utilizados en castellano y portugués}

\begin{tabular}{|c|c|c|}
\hline Autor/es & $\begin{array}{c}\text { Año de } \\
\text { publicación }\end{array}$ & Título \\
\hline Armas, T. de y Venegas, C. & 2016 & $\begin{array}{l}\text { Patriarcado y capitalismo académico. La reproducción de las } \\
\text { violencias }\end{array}$ \\
\hline Azevedo, Mário Luiz Neves de & 2015 & $\begin{array}{l}\text { Transnacionalização e mercadorização da educação superior: } \\
\text { examinando alguns efeitos colaterais do capitalismo acadêmico } \\
\text { (sem riscos) no Brasil - A expansão privado-mercantil }\end{array}$ \\
\hline Briggs, D. & 2017 & $\begin{array}{l}\text { Los siete pecados del capitalismo académico y el crimen } \\
\text { de la ciencia: deconstruyendo la ideología de la "ciencia } \\
\text { criminológica" en España }\end{array}$ \\
\hline Corral, S. & 2015 & $\begin{array}{l}\text { El capitalismo académico: gestión del conocimiento y perfil de } \\
\text { egreso en la Universidad Autónoma de Ciudad Juárez }\end{array}$ \\
\hline \multirow{3}{*}{ Díez, E. } & \multirow{3}{*}{2009} & $\begin{array}{l}\text { El capitalismo académico: la reforma universitaria europea en } \\
\text { el contexto de la globalización }\end{array}$ \\
\hline & & El Plan Bolonia. Capitalismo académico superior \\
\hline & & El capitalismo académico y el Plan Bolonia \\
\hline Fernández, E. & 2009 & $\begin{array}{l}\text { El sistema-mundo del capitalismo académico: procesos de } \\
\text { consolidación de la universidad emprendedora }\end{array}$ \\
\hline $\begin{array}{l}\text { Fernández, E., Rodríguez, H. } \\
\text { y Rodríguez, M. }\end{array}$ & 2010 & $\begin{array}{l}\text { La formación inicial de profesionales de la educación: un } \\
\text { análisis crítico de los nuevos planes de estudio en el contexto } \\
\text { del "capitalismo académico" }\end{array}$ \\
\hline Fernández, R. & 2011 & $\begin{array}{l}\text { Capitalismo académico en la educación superior chilena } \\
\text { ¿Modelo exitoso para el desarrollo de la ciencia? Análisis de } \\
\text { las tendencias en la productividad científica en investigación } \\
\text { durante la última década }\end{array}$ \\
\hline Fioreze, C. & 2017 & $\begin{array}{l}\text { O modelo comunitário de universidade e o tensionamento público- } \\
\text { privado: entre o capitalismo acadêmico e o compromisso social }\end{array}$ \\
\hline \multirow[t]{2}{*}{ Ibarra, E. } & 2005 & $\begin{array}{l}\text { "Capitalismo académico" en los márgenes: notas sobre la } \\
\text { naturaleza de las transformaciones recientes de las universidades } \\
\text { mexicanas }\end{array}$ \\
\hline & 2003 & $\begin{array}{l}\text { Reseña de libro: Capitalismo académico y globalización: la } \\
\text { universidad reinventada }\end{array}$ \\
\hline Krotz, E. & 2012 & $\begin{array}{l}\text { Ciberespacio, ciudadanía, capitalismo académico: cotidianidad } \\
\text { estudiantil y enseñanza de la antropología }\end{array}$ \\
\hline
\end{tabular}

\section{Anexo 2}

Deem, 2001; Hanley, 2005; Johnson y Hirt, 2011; Kauppinen, 2012, 2013a, 2013b; Kauppinen y Kaidesoja, 2014; Leslie, Rhoades

Literatura sobre capitalismo académico en inglés y Oaxaca, 1999; Metcalfe, 2016;

Münch, 2014; Paasi, 2013, 2015,

2016; Rhoades y Slaughter, 2004;

Rhoades y Torres-Olave, 2015;

Slaughter y Leslie, 2001; Slaughter y Rhoades, 2004
Choi, 2010; Enders, 2001; Glenna et al., 2007; González, Martínez y Ordu, 2013; Hoffman, 2011; Mars y Rhoades, 2012; Mendoza, 2012, 2016; Mendoza y Berger, 2008; Mendoza, Kuntz y Berger, 2016; Renault, 2006; Rhoades, 2005; Rhoades y Slaughter, 1997; Welsh et al., 2008; Ylijoki, 2003 
Capitalismo académico: distinciones conceptuales y procesos contradictorios a propósito del caso chileno

Literatura sobre capitalismo académico en castellano y portugués
De Armas y Venegas, 2015; Azevedo, 2015; Briggs, 2017; Díez, 2009a, 2009b, 2009c; Fernández, 2009, 2011; Ibarra, 2005
Corral, 2015; Fernández, Rodríguez y Rodríguez, 2010; Fioreze, 2017; Ibarra, 2003; Krotz, 2012

\begin{abstract}
Academic capitalism: conceptual distinctions and contradictory processes in reference to the Chilean case
\end{abstract}

INTRODUCTION. The present article reviews the specialized literature on the theory of academic capitalism. It seek to specify and develop its basic concepts, and uses them to analyze the Chilean case, both from the point of view of the political economy of its national system of Higher Education and the transformations that universities are undergoing in the direction of entrepreneurial behavior and managerialism. METHOD. A bibliometric analysis of the specialized literature on academic capitalism in English, Spanish, and Portuguese is carried out and then a content analysis is performed with the objective of identifying the different understandings of academic capitalism. RESULTS. Two definitions of academic capitalism are identified: one that focuses on changes at the level of the political and economic systems and the other that revolves around transformations at the organizational level. DISCUSSION. Building upon this basis, an analytical framework is proposed for the study of academic capitalism, distinguishing between political economy processes (marketization, privatization, commercialization and financialization) and their organizational effects (university entrepreneurialism and managerialism). Later, this framework is used to study the Higher Education reform carried out in Chile between the years 2014 and 2018, and aimed at reversing or mitigating the advanced degree of privatism of its Higher Education system. The transformations experienced by academic capitalism as a result of this reform are identified, leading to the conclusion that the outcomes of this process are limited and in some cases ambiguous, and might even cause unanticipated effects, contrary to the objective sought. The article concludes with a brief summary and possible future research lines.

Keywords: Higher Education, Universities, Postsecondary Education as a field of study, Educational objectives.

\title{
Résumé
}

Capitalisme académique: distinctions conceptuelles et processus contradictoires. Le cas chilien

INTRODUCTION. Cet article passe en revue la littérature sur la théorie du capitalisme académique, cherche à clarifiant et développant ses concepts de base. Puis, on utilise ce conceptualization pour analyser le cas chilien, même du point de vue de l'économie politique de son système national d'enseignement supérieur et du les transformations que subissent les universités vers un comportement entrepreneurial et une gestion managériale. MÉTHODE. Le article réalise une 
analyse bibliométrique de la littérature spécialisée sur le capitalisme académique en anglais, espagnol et portugais, il est effectuée puis une analyse de contenu réalisée dans le but d'identifier les différentes compréhensions du capitalisme académique. RÉSULTATS. Le article identifie deux définitions du capitalisme académique: l'une axée sur les changements au niveau de l'économie politique des systèmes et l'autre sur les transformations au niveau organisationnel. DISCUSSION. Sur cette base, un cadre analytique est proposé pour étudier le capitalisme académique en distinguant les processus d'économie politique (marketing, privatisation, marchandisation, commercialisation et financiarisation) et leurs effets organisationnels (université entrepreneuriale et gestion managériale). Ce cadre est utilisé pour étudier la réforme de l'enseignement supérieur tentée au Chili entre 2014 et 2018, visant à inverser ou à atténuer le degré avancé de privatisation de son système, et identifie les transformations subies par le capitalisme académique à la suite de cette réforme, concluant que les résultats à cet égard sont limités et, dans certains cas ambigus, peuvant même entraîner des effets imprévus et contraires à l'objectif cherché. L'article se termine par un résumé et les pistes de possibles recherches futures dans ce domaine.

Mots-clés: Enseignement supérieur, Universités, Enseignement postsecondaire comme domaine d'études, Objectifs éducatifs.

\section{Perfil profesional de los autores}

\section{José Joaquín Brunner}

Doctor en Sociología por la Universidad de Leiden, Holanda. Profesor titular de la Universidad Diego Portales, Chile, y director de la Cátedra UNESCO de Políticas Comparadas de Educación Superior. Sus líneas de investigación giran en torno a la economía política y gobernanza de la educación superior, análisis de sistemas y políticas comparadas.

Correo electrónico de contacto: josejoaquin.brunner@gmail.com

\section{Liliana Pedraja-Rejas}

Doctora en Ciencias de la Educación por la Pontificia Universidad Católica de Chile. Profesora titular de la Universidad de Tarapacá, Chile. Sus líneas de investigación se centran en educación superior, calidad, liderazgo, gestión institucional y estrategia.

Correo electrónico de contacto: lpedraja.rejas@gmail.com

\section{Julio Labraña Vargas (autor de contacto)}

Doctor en Filosofía por la Universidad Witten/Herdecke, Alemania. Investigador asociado de la Universidad Diego Portales, Chile, y coordinador ejecutivo de la Cátedra UNESCO de Políticas Comparadas de Educación Superior. Sus líneas de investigación se centran en la sociología de la educación superior, la historia de la educación y los cambios discursivos.

Correo electrónico de contacto: julio.labrana@mail.udp.cl

Dirección para la correspondencia: Centro de Políticas Comparadas de Educación, Universidad Diego Portales. Avenida Ejército, 260, Santiago, Chile. 